
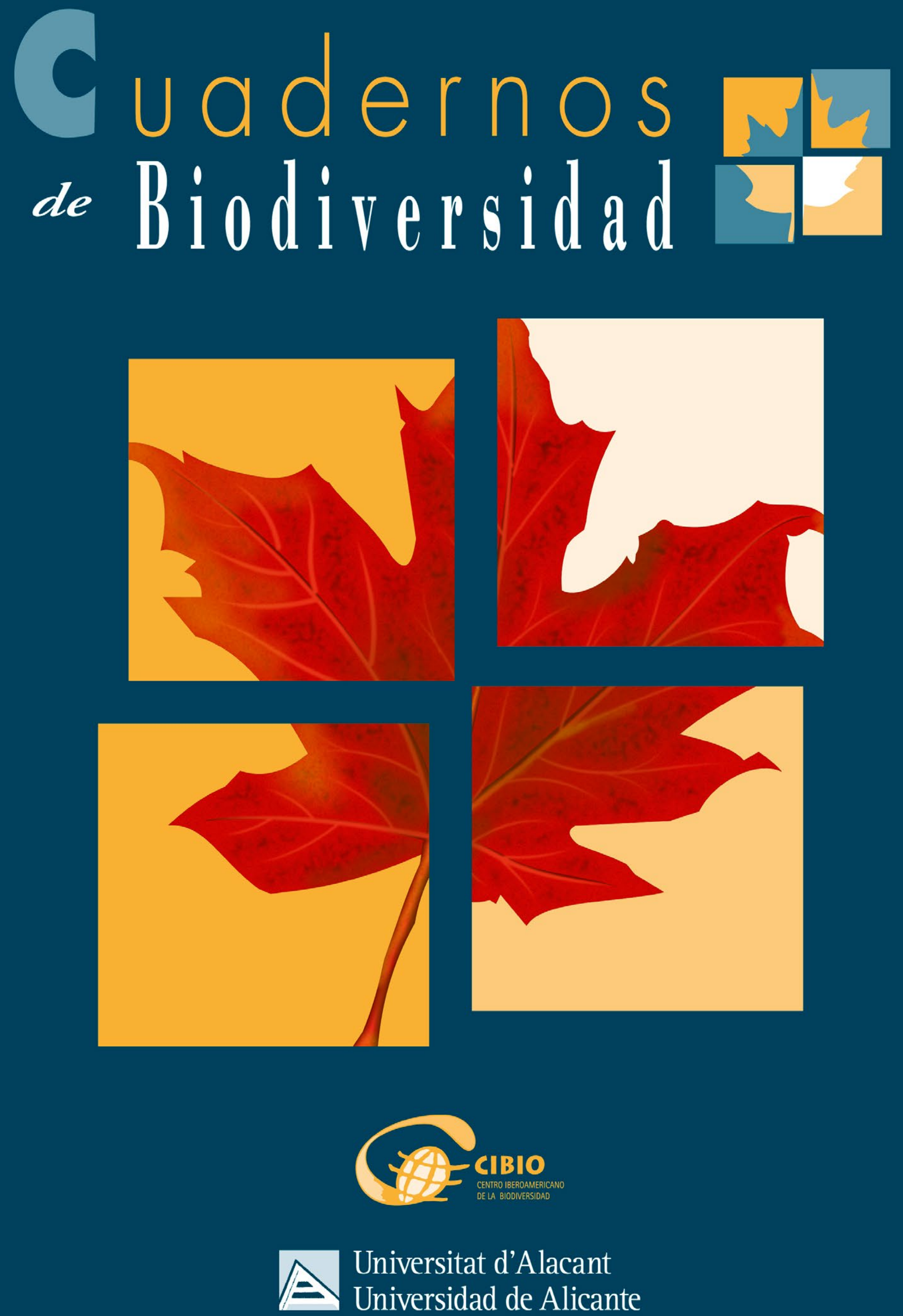


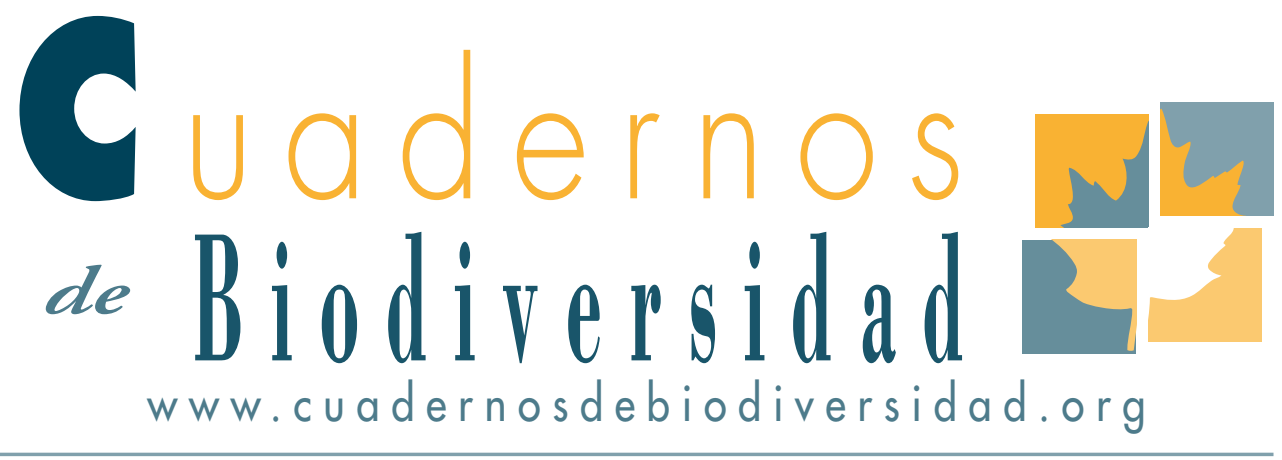

\title{
La Estación Biológica de Torretes (Ilbi, Alicante): un espacio para la conservación de odonatos
}

\section{The Biological Station Torretes (Ilbi, Alicante): a space for the conservation of odonates}

\section{Palomo ${ }^{1}$, C. Quirce $^{2}$, E. Galante 3}

\author{
i Alumna Máster universitario en Gestión y Restauración del Medio Natural. Universidad \\ De Alicante. \\ 2 Asociación Española de Entomología \\ 3 Centro Iberoamericano de la Biodiversidad (CIBIO). Universidad de Alicante. (galante@ua.es)
}

\section{RESUMEN}

Los odonatos son indicadores biológicos de la calidad de los medios acuáticos, ya que requieren, en la mayoría de los casos, aguas limpias y bien oxigenadas para desarrollar sus etapas larvarias. Muchas especies han sido incluidas en la Lista Roja de especies amenazadas. Se ha llevado a cabo un estudio de la fauna de Odonatos en la Estación Biológica Torretes - Jardín Botánico de la Universidad de Alicante (Ibi, Alicante), un espacio de bosque mediterráneo de 53 hectáreas, que está gestionado por el Instituto de Investigación CIBIO de la Universidad de Alicante. Este centro está destinado a investigar y difundir el valor de la biodiversidad y la conservación. Se han estudiado diversos puntos de agua permanentes con el objetivo de determinar áreas de conservación de la biodiversidad. En la estación biológica se regis- traron 16 especies de odonatos de los 28 citadas en ambientes naturales para la provincia de Alicante, lo que resaltan la importancia de este espacio natural en la conservación de estos insectos.

Pallabras clave: Odonatos - Bioindicador - Agua embalsada - Estación Biológica

\section{ABSTRACT}

Odonates are biological indicators of the aquatic environments quality, because them require, in most cases, clean and well oxygenated waters to develop their larval stages. Many species have been included in the Red List of threatened species for their vulnerability to contaminated environments. A study of odonata fauna was carried out in several permanent 
water points in the Biological Station - Botanical Garden of Torretes (Ibi, Alicante), a Mediterranean forest space of 53 hectares, managed by the Research Institute CIBIO of the University of Alicante. This center aims to investigate and disseminate the value of biodiversity and conservation. In the biological station was collected 16 species Odonates of the 28 reported in natural environments for the Alicante province, which highlight the importance of this natural space in the conservation and biodiversity of these insects.

Key words: Odonata - Bioindicator - Reserved water - Biological Station

\section{INTRODUCTION}

Los Odonatos son uno de los grupos más antiguos de insectos, cuya existencia se conoce desde el Pérmico (Torralba-Burrial, 2015). Constituyen un orden cuya diversidad y abundancia está ligada a las zonas húmedas y cursos de agua donde se desarrollan sus fases larvarias que son siempre acuáticas, mientras que los adultos son voladores en su entorno.

Son insectos depredadores, conocidos como libélulas (Suborden Anisoptera) y caballitos del diablo (Suborden Zygoptera), que vemos volar con frecuencia desde la primavera hasta entrado el otoño en torno a lagunas, balsas, ríos y arroyos, siempre que su agua esté suficientemente oxigenada y libre de contaminantes, razón por la cual son considerados en general como buenos bioindicadores del estado de conservación de los medios acuáticos (Valladares et al., 2004, Oertli, 2008).

La contaminación creciente y la alteración de los ecosistemas de agua dulce, les ha afectado gravemente en muchos lugares del mundo, hasta el punto de que muchas de sus especies se encuentran amenazadas, siendo uno de los grupos con mayor presencia en catálogos y listados de especies amenazadas (Valladares et al., 2004). De las más de 5600 especies de odonatos descritas hasta el momento en el mundo, 176 han sido incluidas en alguna categoría de amenaza en la Lista Roja de la UICN (TorralbaBurrial, 2015).
En el caso de la península ibérica, de las 79 especies presentes, 18 se encuentran incluidas en el Libro Rojo de los Invertebrados de España (Verdú \& Galante, 2006).

La Lista Roja recoge un total de 51 especies de odonatos presentes en la península ibérica, la mayoría de ellas tienen la categoría LC (procupación menor), tres de ellas: Cordulegaster bidentata, Gomphus graslinii y Onychogomphus costae están en la categoría de casi amenazadas (NT) y Macromia splendens está catalogada como especie vulnerable (VU)

En el caso de la Comunidad Valenciana, la Orden del 1 de diciembre de 2006, de la Conselleria de Territorio y Vivienda, amplía el Catálogo Valenciano de Especies de Fauna Amenazada con la inclusión de diez nuevas especies dentro de la categoría de vulnerables. Entre estos diez taxones figuran tres odonatos con presencia escasa en la Comunidad Valenciana o por estar incluidos en catálogos europeos de protección. Estas tres especies son Coenagrion mercuriale, Gomphus graslinii y Oxygastra curtisii. (Voluntariat parotets, 2013)

Gracias a los muestreos llevados a cabo por el grupo de "Voluntariat parotets" se conoce la presencia de dos especies vulnerables en la Comunidad Valenciana como son Gomphus graslinii y Oxygastra curtisii y se crea la primera reserva de fauna para libélulas de toda la Comunitat Valenciana, en la comarca de la Canal del Navarrés (Valencia) en el año 2010. (Voluntariat parotets, 2013)

En el levante ibérico, como ocurre en toda la cuenca mediterránea, la actividad humana de explotación agropecuaria del territorio se ha llevado a cabo desde hace miles de años y ha sido un factor decisivo en la modelización del característico paisaje mediterráneo heterogéneo y dinámico que observamos y que está conformado por una elevada diversidad de hábitats (Blanco et al., 1997; García Antón et al., 2002, San Miguel et al., 2012). Es precisamente esta diversidad espacial la responsable de la alta diversidad y endemicidad entomológica que encontramos en la cuenca mediterránea (Galante, 2002, Galante \& Marcos-García, 2013). 
Es éste el paisaje que caracteriza la provincia de Alicante, donde encontramos grandes áreas de producción agrícola que alternan con zonas de monte mediterráneo. Podemos encontrar pequeños ríos y arroyos sometidos a un elevado estiaje y algunas balsas de riego y puntos diversos de acumulación de agua que se suceden a lo largo de toda su extensión.

Con el fin de estudiar este grupo de insectos y analizar su estado de conservación, se llevó a cabo éste estudio que está dirigido a evaluar el potencial como posible reserva de fauna para libélulas en la Estación Biológica/Jardín Botánico de Torretes, un espacio natural gestionado por el instituto de investigación CIBIO (Centro Iberoamericano de la Biodiversidad) de la Universidad de Alicante.

La Estación Biológica Torretes/Jardín Botánico de la Universidad de Alicante se ubica en el interior de la provincia de Alicante, en el término municipal de Ibi. Es un espacio dedicado a la investigación y a la de conservación de flora y fauna.

Se trata de un paisaje típico de monte mediterráneo, con alta diversidad florística y de hábitats (Ríos \& Martínez-Francés, 2007) y donde existen pequeñas zonas de retención de agua permanente, que conforman hábitats de interés para albergar poblaciones de insectos ligados al medio acuático.

Teniendo en cuenta las características de este espacio, se consideró que podría favorecer el asentamiento de poblaciones de odonatos, facilitando el reposo de las especies migratorias, y creando las condiciones favorables para la reproducción y el desarrollo larvario, contribuyendo de este modo a la conservación de los odonatos ibéricos.

Con este fin se plantearon los siguientes objetivos:

1. Conocer la diversidad de odonatos del área y llevar a cabo un inventario faunístico de la zona.

2. Analizar los requerimientos ecológicos de las especies registradas.

3. Estudiar las interacciones y relaciones con los hábitats.

4. Proponer, en su caso, medidas de gestión y de mejora de hábitats.

\section{MATERIAL Y MÉTODOS}

La Estación Biológica/Jardín Botánico de Torretes (Figura 1) se encuentra al norte de la provincia de Alicante, en el término municipal de Ibi, situada entre los 900-1100 metros de altitud. Fue creada el 29 de marzo de 2003 mediante un acuerdo entre la Universidad de Alicante y el Ayuntamiento de Ibi (Alicante), estando adscrita al Centro Iberoamericano de la Biodiversidad (CIBIO), un instituto de investigación de la Universidad de Alicante.

Se trata de una finca de secano denominada "Les Torretes" situada en la vertiente sur del Parque Natural del Carrascal de la Font Roja (Alicante) a 950 m, en el NE del término municipal de Ibi. El clima es mediterráneo con rangos de precipitación media anual de entre $843 \mathrm{~mm}$ en las cumbres del área montañosa norte (Menejador, $1352 \mathrm{~m}$ ) y los $402 \mathrm{~mm}$ en la vertiente sur (Ibi, $816 \mathrm{~m}$ ) (Ríos \& Martínez-Francés, 2007). El patrón de lluvias es Otoño-Invierno-Primavera-Verano, con un pico en octubre cuando la mitad de las precipitaciones totales se dan en forma de lluvias torrenciales (Cuba et al., 2008)

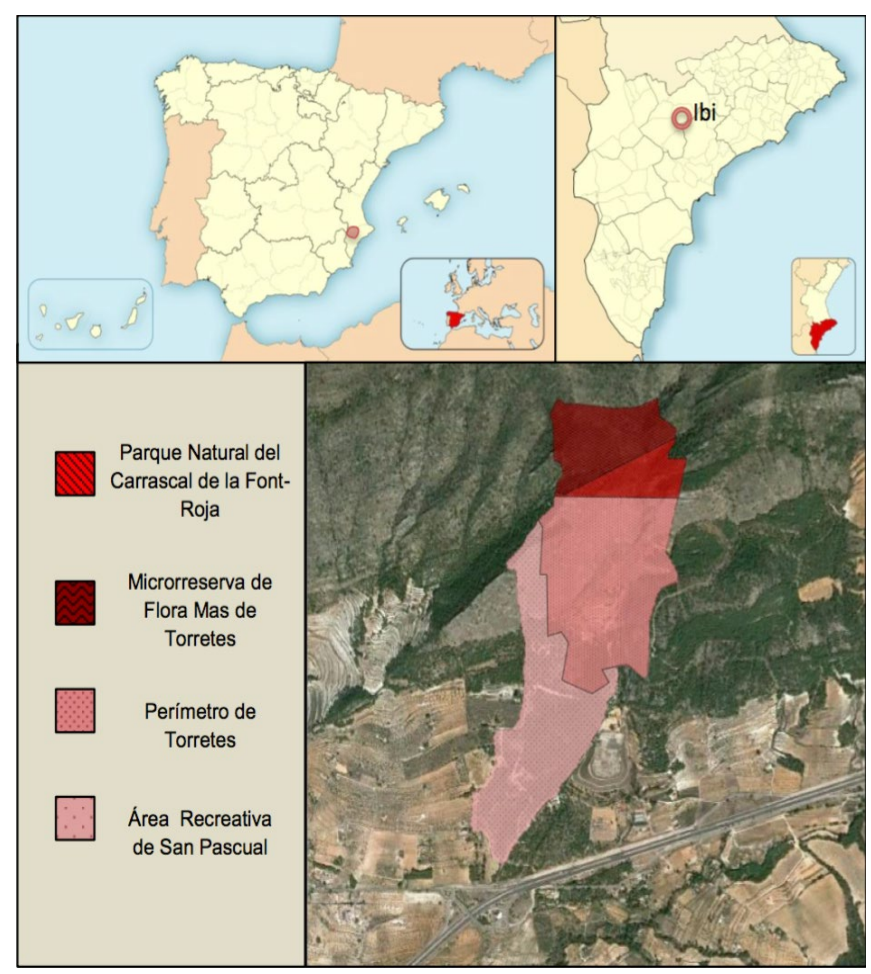

Figura 1: Áreas protegidas: Delimitación 
Este espacio cuenta con una superficie de 53 hectáreas, de las que su mayor parte corresponden a superficie forestal. Se puede encontrar matorral esclerófilo, lastonares, sabinares de roquedo, pinar de pino carrasco y alguna pequeña superficie de espartal, siendo su superficie agraria útil de 9 hectáreas. Existe una zona de antiguos cultivos de secano de cereal, principalmente almendro y olivo (Cuba et al., 2008).

En el 2010 se designaron 20 hectáreas de la Finca Torretes como Microrreserva de Flora de la Generalitat Valenciana, lo que supuso el reconocimiento de la riqueza botánica de este enclave. Además, en 2010 se designaron otras 35,6 hectáreas de la Estación como Reserva de Fauna (Orden 18/2010 de la Consellería de Agricultura, Medio Ambiente, Cambio Climático y Desarrollo Rural, Generalitat Valenciana).

Sobre estas terrazas de antiguos cultivos se han asentado colecciones botánicas, lo que ha permitido que desde el año 2012 haya sido reconocido como Jardín Etnobotánico por la Asociación Ibero Macaronésica de Jardines Botánicos (AIMJB), convirtiéndose en el segundo Jardín Botánico de la Comunidad Valenciana y el primero y único de la provincia de Alicante (Marcos \& Micó, 2012).

La Estación Biológica de Torretes alberga 3 balsas (Figura 2) y dos depósitos de agua (potable y pluviales). Las 3 balsas fueron construidas hace aproximadamente diez años y han sido uno de los hábitats seleccionados para este estudio. Estas balsas mantienen en sus márgenes y medio acuático, importantes formaciones vegetales que constituyen un elemento esencial en las actividades de caza y reproducción de los odonatos adultos.

- Balsa no 1 Zona de cría y desove de anfibios: engloba 4 pozas naturales de pequeño tamaño que se llenan de agua de lluvia. El agua discurre a través de las pozas naturales, diversificando los espacios para la fauna acuática. Esta balsa alberga numerosas poblaciones de renacuajos. 38³8'10.40” N 0³2'13.67”O.
- Balsa no 2 Sauceda y Estanque de Carlos Vicioso: es la balsa de mayor tamaño, cuenta con unos 36 metros cuadrados, y está rodeada de juncos, sauces, rosas y otras especies herbáceas. Esta balsa está impermeabilizada para evitar pérdidas del agua en un terreno extremadamente árido en verano, presenta en algunos momentos del año altos niveles de eutrofización. 38³8'10.66” N 0³2'13.08” O.

\section{- Balsa no 3 Barranco de Enric Valor y Pozo} de Paracelso: se encuentra en el punto con más altitud de la Estación. Es de un tamaño intermedio entras las otras dos balsas y alberga abundante vegetación acuática. 38³8’14.04” N 0³2'11.94” O.

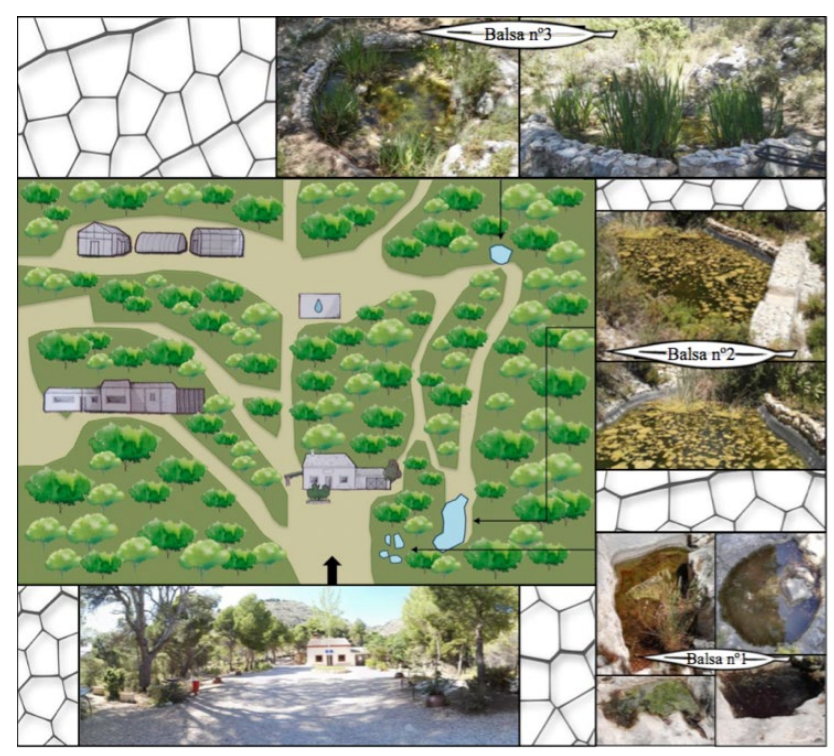

Figura 2. Balsas de muestreo de la Estación Biológica/ Jardín Botánico de Torretes

Para conocer las especies de odonatos que habitan en la Estación Biológica/Jardín Botánico de Torretes, y poder hacer una comparación con la fauna conocida de odonatos de la provincia de Alicante, se ha revisado el material entomológico perteneciente a la Colección Entomológica de la Universidad de Alicante (CEUA) que está depositada y custodiada en el Centro Iberoamericano de la Biodiversidad de la Universidad de Alicante (CIBIO). Asimismo se han consultado las bases de datos del Banco de Datos de Biodiversidad de la Comunidad Valenciana (BDDB) con el fin de obtener un inventario actualizado de la zona. 
Las balsas elegidas (Figura 2) para realizar el muestreo fueron visitadas a lo largo de los meses de marzo a junio de 2016, abarcando de este modo la primavera y el inicio del verano, llevándose a cabo el muestreo con una periodicidad de 20 días. Se eligieron siempre para los muestreos días con condiciones soleadas y cielo despejado, para favorecer el posible avistamiento de ejemplares. En cada muestreo se anotaban tanto las condiciones atmosféricas del día (Tabla 1) como las condiciones y estado de cada balsa (Tabla 2).

La extraordinaria sequía imperante durante el ańo de estudio tanto a principios del otońo como en invierno, imposibilitó el poder llevar a cabo registros de vuelo de algunas especies en esos periodos.

En este estudio se ha utilizado como método de muestreo el conteo visual mensual en las 3 balsas. Se han realizado 3 repeticiones de conteo de 30 minutos de duración en cada una.
Para la identificación de las especies se ha seguido a Baixeras et al. (2005) y Dijkstra, \& Lewington (2006). La identificación se ha llevado a cabo tanto mediante registros fotográficos, como mediante captura del ejemplar con posterior suelta y sólo en caso necesario, se trasladaba el ejemplar al laboratorio para su correcta identificación.

Para la captura de adultos se ha utilizado una manga entomológica con un aro de $40 \mathrm{~cm}$ de diámetro y un mango de 1 metro de longitud. Se capturaban directamente los ejemplares y también se batió la vegetación de la zona, donde permanecen resguardadas muchas especies.

En cada uno de los muestreos se anotaba en una hoja de registro: el número de balsa, la fecha, el estado meteorológico del día, la hora, el número de réplica y el $\mathrm{pH}$.

Se registró también la temperatura del agua mediante termómetro digital, y el $\mathrm{pH}$. Ambas variables son importantes ya que indican la calidad del agua y de ellas depende la posible existencia de ninfas y adultos de odonatos.

\begin{tabular}{|c|c|c|c|c|c|c|}
\hline & $\begin{array}{c}\text { Temperatura } \\
\left({ }^{\circ} \mathbf{C}\right)\end{array}$ & $\begin{array}{c}\text { Humedad } \\
(\%)\end{array}$ & $\begin{array}{c}\text { Presión } \\
\text { (hPa) }\end{array}$ & $\begin{array}{l}\text { Precipitaciones } \\
\text { (mm) }\end{array}$ & $\begin{array}{l}\text { Viento } \\
(\mathbf{k m} / \mathbf{h})\end{array}$ & $\begin{array}{c}\text { Días de } \\
\text { sequía }(*)\end{array}$ \\
\hline \multirow{2}{*}{$\begin{array}{c}\text { Meses de } \\
\text { muestreo } \\
\text { (marzo- } \\
\text { junio) }\end{array}$} & 32.5 & 91 & 1019 & \multirow{2}{*}{0.0} & \multirow{2}{*}{39} & 34 \\
\hline & 12.1 & 9 & 1001 & & & $\begin{array}{l}(*) \text { días } \\
\text { enteros }\end{array}$ \\
\hline \multirow{2}{*}{$\begin{array}{c}\text { Año } \\
(2016)\end{array}$} & 32.5 & 95 & 1035 & \multirow{2}{*}{91.6} & \multirow{2}{*}{68} & \\
\hline & -2.2 & 9 & 991 & & & $\mathrm{~mm}$. \\
\hline
\end{tabular}

Tabla 1. Condiciones atmosféricas a lo largo del año 2016, incluye los meses de muestreo en la Estación Biológica de Torretes. En rojo se muestra la media de las máximas temperaturas, humedad y presión y en azul la media de las mismas.

\begin{tabular}{|c|c|c|c|}
\hline $\mathbf{N}^{\mathbf{0}}$ de Balsa & \multicolumn{2}{|c|}{ Temperatura $\left({ }^{\circ} \mathrm{C}\right)$} & pH \\
\hline Balsa n $^{\mathbf{0}} \mathbf{1}$ & $12,5^{\circ} \mathrm{C}$ & $20,1^{\circ} \mathrm{C}$ & 8,29 \\
\hline Balsa n $^{\mathbf{0}} \mathbf{2}$ & $17,8^{\circ} \mathrm{C}$ & $25,6^{\circ} \mathrm{C}$ & 8,19 \\
\hline Balsa n $^{\mathbf{0}} \mathbf{C}$ & $15,7^{\circ} \mathrm{C}$ & $22,3^{\circ} \mathrm{C}$ & 7,12 \\
\hline
\end{tabular}

Tabla 2. Condiciones del medio acuático de cada balsa. Se expresa la temperatura mínima y máxima registrada en cada balsa durante los meses de muestreo así como el pH. 


\section{RESULTADOS Y DISCUSIÓN}

Del total de 58 especies de odonatos citadas en la Comunidad Valenciana (Baixeras et al., 2005) al menos 28 se han registrado en la provincia de Alicante donde existen condiciones favorables para su desarrollo. De ellas al menos 16 especies se han observado en la Estación Biológica/Jardín Botánico de Torretes:

\begin{tabular}{|c|c|c|}
\hline ESPECIE & SUBORDEN & FAMILIA \\
\hline $\begin{array}{l}\text { Lestes viridis (Van } \\
\text { der Linden, 1825) }\end{array}$ & Zygoptera & Lestidae \\
\hline $\begin{array}{l}\text { Pyrrhosoma } \\
\text { nymphula (Sulzer, } \\
1776 \text { ) }\end{array}$ & Zygoptera & Coenagrionidae \\
\hline $\begin{array}{l}\text { Sympecma fusca } \\
\text { (Van der Linden, } \\
1820 \text { ) }\end{array}$ & Zygoptera & Lestidae \\
\hline $\begin{array}{l}\text { Aeshna mixta } \\
\text { (Latreille, 1805) }\end{array}$ & Anisoptera & Aeshnidae \\
\hline $\begin{array}{l}\text { Anax imperator } \\
\text { (Leach, 1815) }\end{array}$ & Anisoptera & Aeshnidae \\
\hline $\begin{array}{l}\text { Anax parthenope } \\
\text { (Selys, 1839) }\end{array}$ & Anisoptera & Aeshnidae \\
\hline $\begin{array}{l}\text { Crocothemis } \\
\text { erythraea (Brullé, } \\
1832 \text { ) }\end{array}$ & Anisoptera & Libellulidae \\
\hline $\begin{array}{l}\text { Libellula depressa } \\
\text { (Linnaeus, 1758) }\end{array}$ & Anisoptera & Libellulidae \\
\hline $\begin{array}{l}\text { Orthetrum brun- } \\
\text { neum (Fonsco- } \\
\text { lombe, 1837) }\end{array}$ & Anisoptera & Libellulidae \\
\hline $\begin{array}{l}\text { Orthetrum cance- } \\
\text { llatum (Linnaeus, } \\
1758 \text { ) }\end{array}$ & Anisoptera & Libellulidae \\
\hline $\begin{array}{l}\text { Orthetrum coeru- } \\
\text { lescens (Fabricius, } \\
1798)\end{array}$ & Anisoptera & Libellulidae \\
\hline $\begin{array}{l}\text { Sympetrum striola- } \\
\text { tum (Charpentier, } \\
1840 \text { ) }\end{array}$ & Anisoptera & Libellulidae \\
\hline $\begin{array}{l}\text { Sympetrum fonsco- } \\
\text { lombii (Selys, 1840) }\end{array}$ & Anisoptera & Libellulidae \\
\hline
\end{tabular}

El suborden Zygoptera se encuentra representado por 4 especies y el suborden Anisoptera por 12 especies. La presencia de algunas especies de Anisoptera puede deberse a la reciente creación de estos espacios con agua permanente de la estación biológica que han sido colonizados progresivamente a lo largo de los últimos diez años.

Las especies de Anisoptera son los odonatos de mayor envergadura y capacidad de vuelo, lo que les permite mayor capacidad de dispersión. Sus especies en general llevan a cabo grandes migraciones que cubren grandes distancias, lo que les permite colonizar con frecuencia nuevos espacios (http://www. migratorydragonflypartnership.org/index/welcome) como es el caso de los nuevos hábitats acuáticos creados en los últimos años en la Estación Biológica/ Jardín Botánico de Torretes.

Por el contrario el bajo número de especies de zigópteros registrados puede deberse a su menor tamaño y menor capacidad de vuelo y dispersión. Además, su hábitat suele estar ligado a la existencia de gran cantidad de vegetación en los márgenes de los cursos de agua donde los adultos pueden reposar y esperar ocultos a sus posibles presas. Todo ello unido a que sus fases larvarias son menos tolerantes a los déficits de oxígeno que en ocasiones se producen en este tipo de charcas y balsas. 
El buen estado de conservación de estos puntos de agua, y el enriquecimiento que se ha producido en su entorno a lo largo del tiempo, han incrementado los refugios para las ninfas de odonatos.

Se ha registrado la presencia de especies de la familia de odonatos Coenagrionidae, consideradas como buenas indicadoras de la calidad ambiental (Briers \& Biggs, 2003). Además es relevante la observación de especies como Libellula depressa (Linnaeus, 1758), cuya primera cita en la provincia de Alicante se produjo en el ańo 2009 en la balsa no 2 de Torretes y desde entonces se han recogido citas de numerosos ejemplares de esta especie a lo largo de años sucesivos.

Los resultados obtenidos y los registros llevados a cabo año tras ańo, han puesto de manifiesto el potencial de la Estación Biológica/Jardín Botánico de Torretes como un área de especial interés para la conservación de odonatos.

Por otra parte, es posible realizar seguimientos anuales de las poblaciones de estas especies y medir las fluctuaciones en composición y abundancia de las todas ellas. De este modo se podrán adoptar las medidas necesarias de gestión y adecuación de los hábitats que tengan en cuenta los requerimientos de las distintas especies, en particular los requerimientos de las fases larvarias.

Todos estos datos indican que la Estación Biológica/Jardín Botánico de Torretes puede convertirse en una reserva de fauna para libélulas.

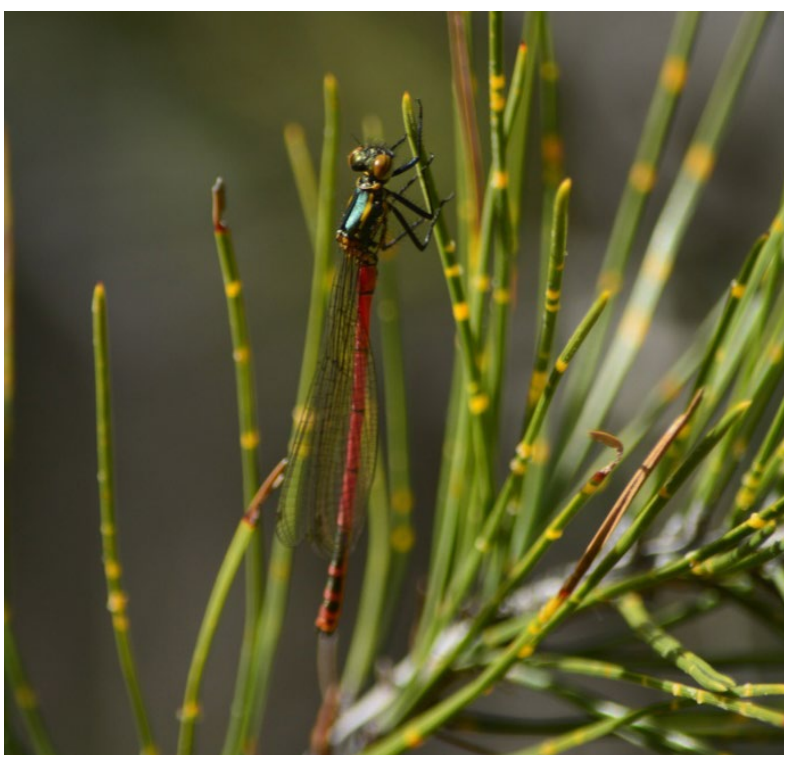

Pyrrhosoma nymphula. Fotografía: M. Palomo

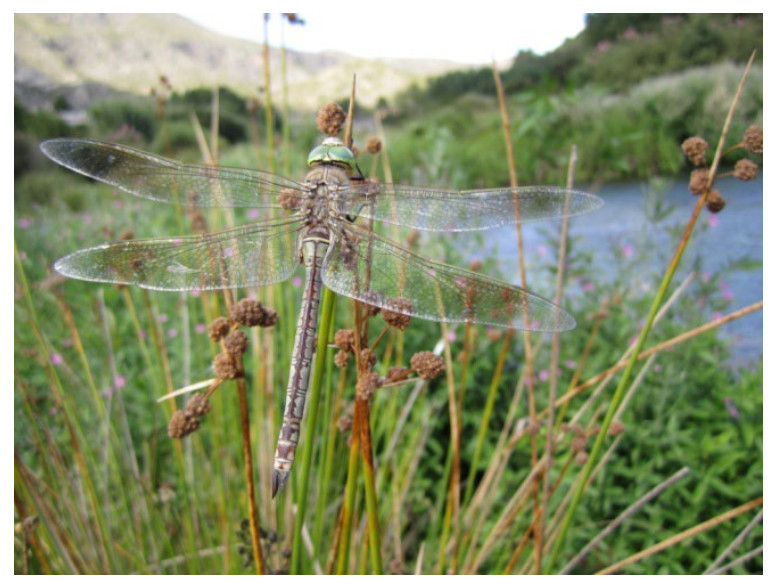

Anax parthenope. Fotografía: C. Quirce

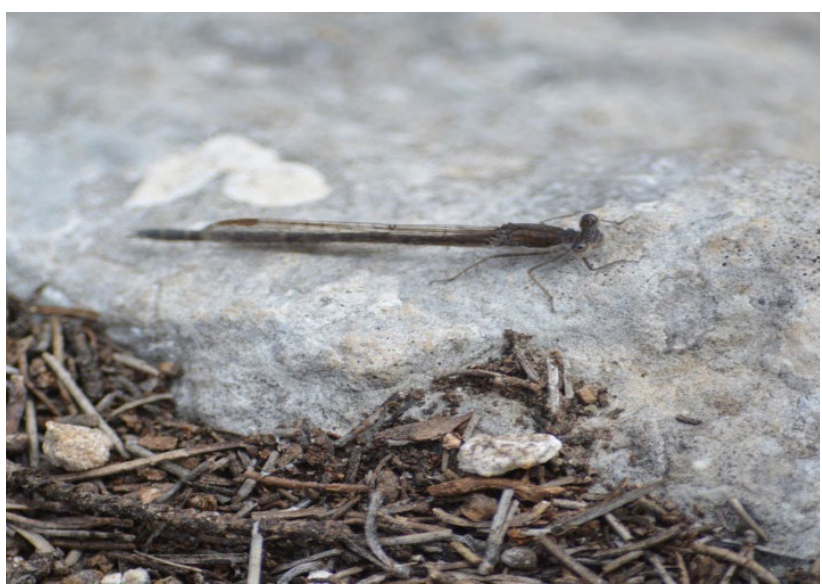

Sympecma fusca. Fotografía: M. Palomo

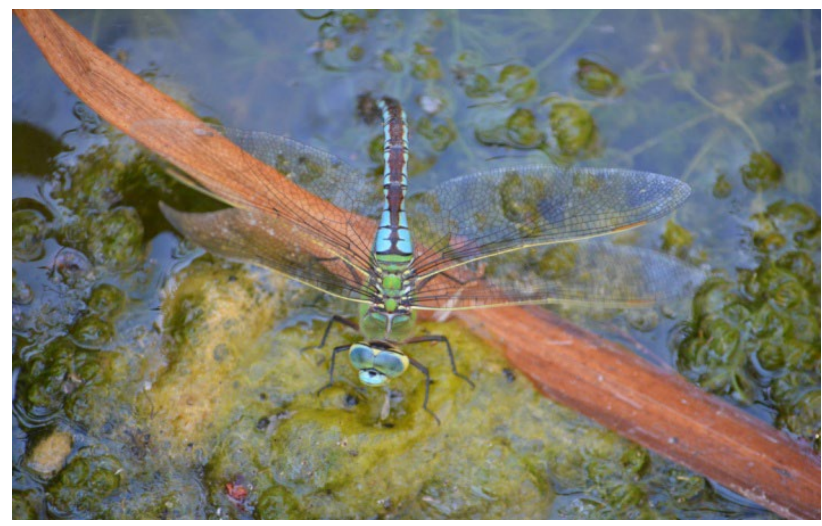

Anax imperator. Fotografía M. Palomo 


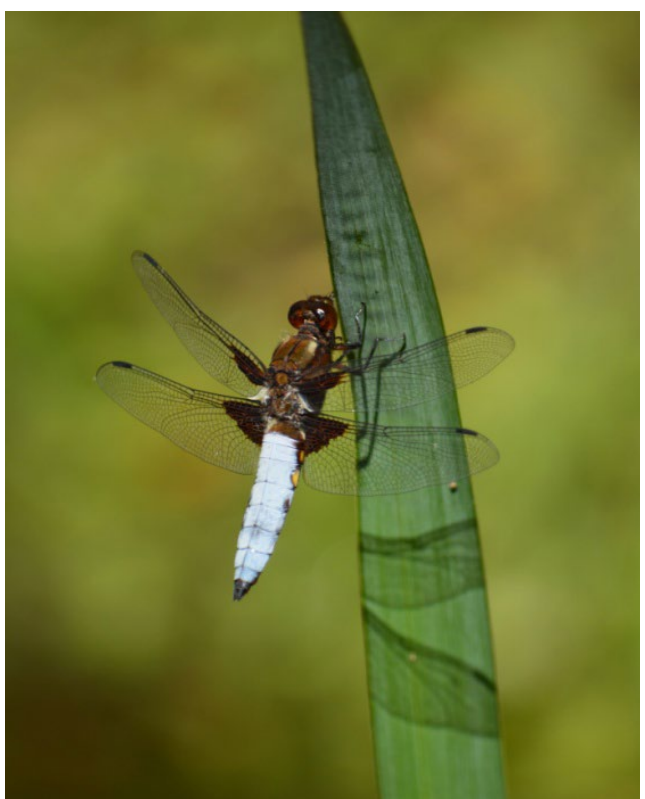

Libellula depressa. Fotografía: M. Palomo

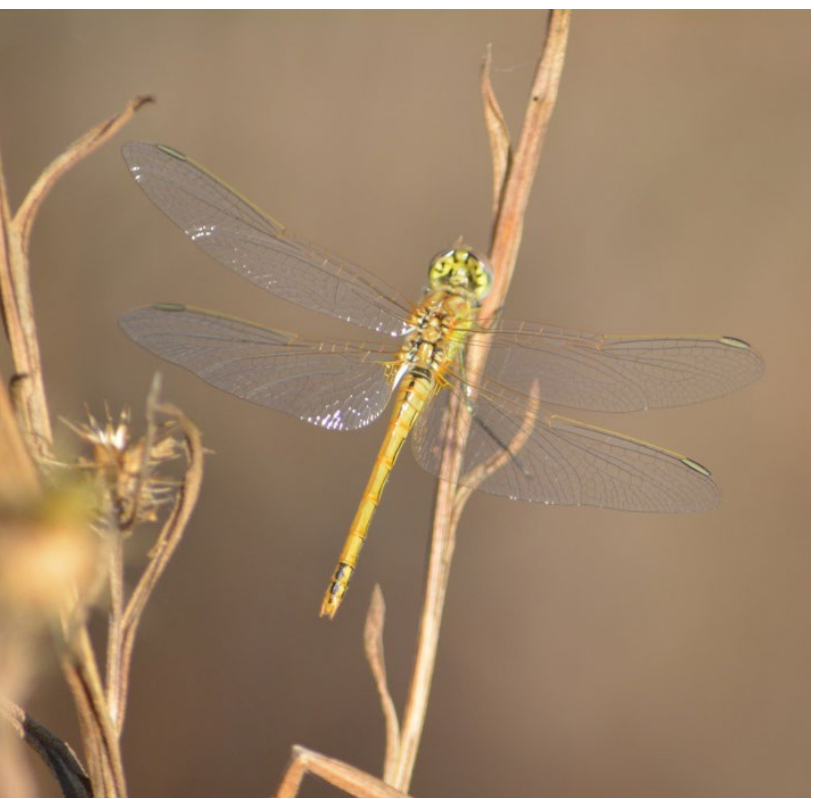

Sympetrum fonscolombii. Fotografía: M. Palomo

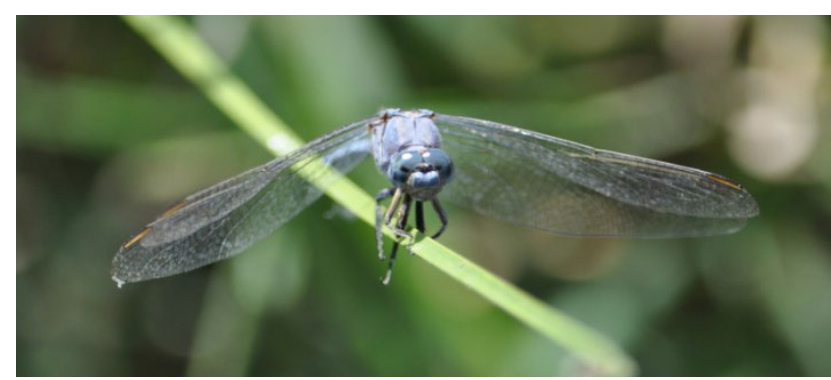

Orthetrum brunneum. Fotografía: M. Palomo

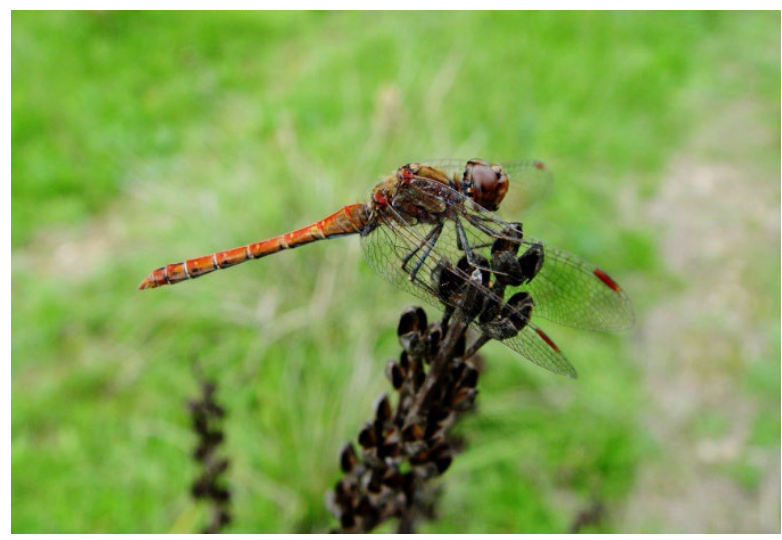

Sympetrum striolatum. Fotografía: E. Galante

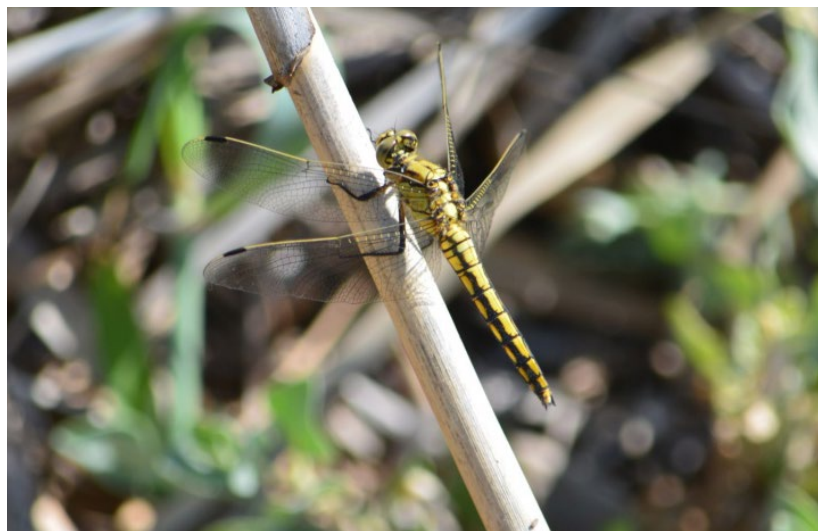

Orthetrum coerulescens. Fotografía M. Palomo

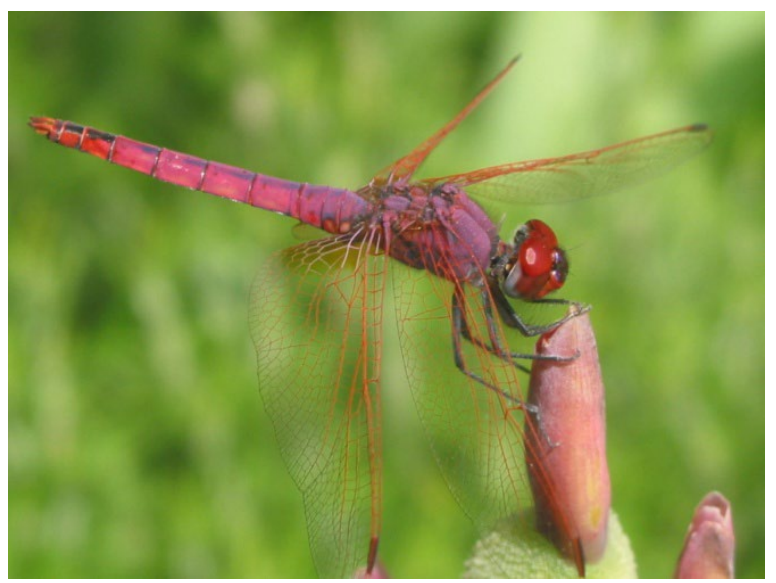

Trithemis annulata. Fotografía: C. Quirce 


\section{REFERENCIAS}

Baixeras, J., Michelena, J.M., González, P., Ocharán, F., Quirce, C., Marcos- Gacía, M.A., Soler, E., Domingo, J., Montagud, S., Gutierrez, A. \& Arles, M. (2005). Les Libèl-lules de la Comunitat Valenciana. Conselleria de Territori i Habitatge. Generalitat Valenciana, Valencia. ISBN 84-482-4248-3

Banco de Datos de Biodiversidad de la Comunidad Valenciana: http://bdb.cma.gva.es/web/acciones.aspx?url=http:// bdb.cma.gva.es/arbol.htm\&logo=http://bdb.cma.gva es/bdb.jpg\&an=http://bdb.cma.gva.es/bdb2.jpg\&gana=UA-16710898-11/

Blanco, E., Casado, M. A., Costa, M., Escribano, R., \& García, M., Génova, M., Gómez, A., Gómez F, Moreno, J.C., Morla, C., Regato, P., Sáinz, H. (1997) Los Bosques Ibéricos. Una Interpretación Geobotánica. Planeta, Madrid, Spain.

Briers, R.A, Biggs, J. (2003). Indicator taxa for the conservation of pond invertebrate diversity. Aquatic Conservación: marine and Freshwater Ecosystems. 13:323-330

Cuba, O., Bañón, C. \& Marcos, M.A. (2008). Los formícidos (Hymenoptera, formicidae) del Parque Natural de la font Roja. Revista per al coneixement dels ecosistemes del Carrascal de la Font Roja. IBERIS. 8-9 pp.

Del Estado, B. O. (1990). Real Decreto 439/1990 por el que se establece el Catálogo Nacional de Especies Amenazadas. Boletín Oficial del Estado, 82, 9468-9471.

Dijkstra, K. D. B., \& Lewington, R. (2006). Field guide to the dragonflies of Britain and Europe: including western Turkey and north-western Africa. British Wildlife Publishing.

Galante, E. (2002). Los Insectos. En J.M. Reyero (editor) La Naturaleza de Espańa: 208-215. Ministerio de Medio Ambiente

Galante, E. \& Marcos-García, M.A. (2013). El bosque mediterráneo ibérico: un mundo manejado y cambiante. En Micó E., M.A marcos-García \& E. Galante (eds) Los Insectos saproxílicos del Parque Nacional de Cabañeros: 11-32. Organismo Autónomo Parques Nacionales. Ministerio del Medio Ambiente. España ISBN: 97884-8014-854-
García-Antón, M., Maldonado Ruiz, J., Morla Juaristi, C., \& Sainz-Ollero, H. (2002). Fitogeografía histórica de la Península Ibérica.La diversidad biológica de España, 45-63.

Marcos, M.A \& Micó, E. (2012). Cuadernos de Biodiversidad. CIBIO. 39:11. 14pp.

Oertli B.,(2008). The use of dragonflies in the assessment and monitoring of aquatic habitats. In: Córdoba-Aguilar A (ed) Dragonflies \& damselflies: model organisms for ecological and evolutionary research. Oxford University Press, Oxford, pp 79-95

Ríos, S. \& Martínez-Francés, V. (2007). El Jardín Etnobotánico de la Estación Biológica de Torretes-Font Roja,CIBIO-Universidad de Alicante. Cuadernos de biodiversidad. No 23 (jun. 2007), p. 21.

San Miguel A., Perea, R., Roig, S. \& Fernández-Olalla, M. (2012). Bosques y matorrales mediterráneos continentales. Ambienta 98: 66-72

Torralba-Burrial, A. (2015). Clase Insecta. Orden Odonata Revista IDE@-SEA 41: 22pp. Accesible en: http://www. sea- entomologia.org/IDE@/revista_41.pdf

Valladares, L.F, García, F., Vega, F.J \& Miguélez, D. (2004). Estudio de la fauna de Odonatos de los humedales de Salburua (Victoria-Gasteiz).

Verdú, J.R. \& Galante, E. (Eds) (2006) Libro Rojo de los Invertebrados de España. Madrid, Ministerio de Medio Ambiente, 248-277.

Verdú, J.R, Numa, C. \& Galante, E. (eds) (2011) Atlas y Libro Rojo de los invertebrados amenazados de España (especie Vulnerables). Madrid, MMARM, 588-594.

Voluntariat parotets (2013). Aportación del Voluntariat Parotets a la conservación y conocimiento de los odonatos en la Comunitat Valenciana. Quaderns de voluntariat i participació social, 1. Valencia. Conselleria d'Infraestructures, Territori i Medi Ambient. Generalitat Valenciana. 48 pp. 
Este número ha recibido una ayuda del Vicerrectorado de Investigación y Transferencia de Conocimiento de la Universidad de Alicante
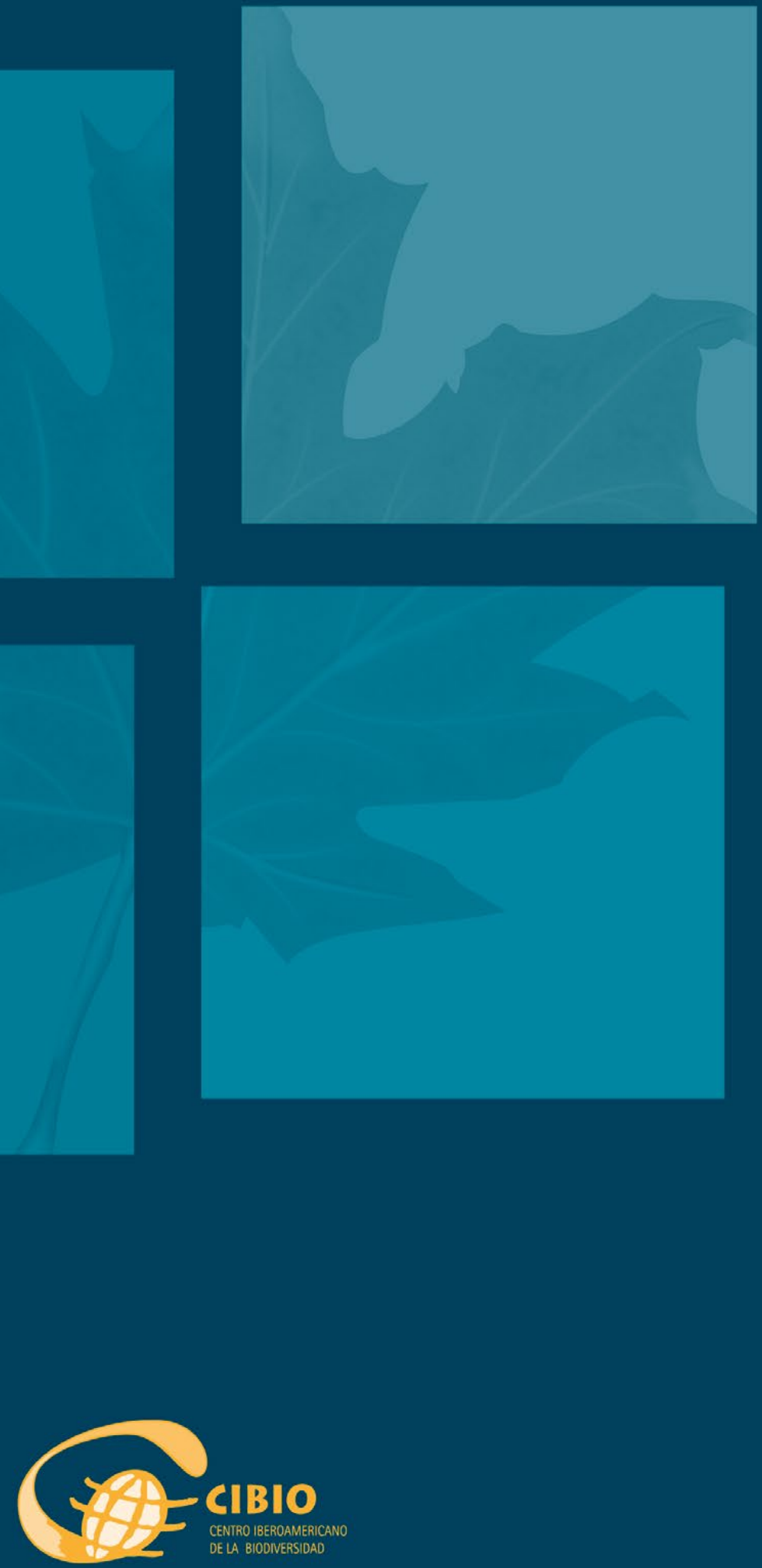

\section{Universitat d'Alacant
Universidad de Alicante}

